\title{
Sequential treatment with afatinib and osimertinib in patients with EGFR mutation-positive non-small-cell lung cancer: an observational study
}

\author{
Maximilian J Hochmair*,1, Alessandro Morabito², Desiree Hao ${ }^{3}$, Cheng-Ta Yang ${ }^{4}$, Ross A \\ Soo $^{5}$, James C-H Yang ${ }^{6}$, Rasim Gucalp ${ }^{7}$, Balazs Halmos ${ }^{7}$, Lara Wang ${ }^{8}$, Amanda \\ Golembesky $^{9}$, Angela Märten ${ }^{10}$ \& Tanja Cufer ${ }^{11}$ \\ ${ }^{1}$ Department of Respiratory \& Critical Care Medicine, Ludwig Boltzmann Institute of COPD \& Respiratory Epidemiology, Otto \\ Wagner Hospital, Sanatoriumstrasse 2, 1140 Vienna, Austria \\ ${ }^{2}$ Thoracic Medical Oncology, Istituto Nazionale Tumori, 'Fondazione G.Pascale'-IRCCS, 80131 Napoli, Italy \\ ${ }^{3}$ Tom Baker Cancer Center, Cummings School of Medicine, University of Calgary, Calgary, Alberta, Canada \\ ${ }^{4}$ Department of Thoracic Medicine, Chang Gung Memorial Hospital, Taoyuan, Taiwan \\ ${ }^{5}$ Department of Haematology-Oncology, National University Hospital, Singapore \\ ${ }^{6}$ Department of Oncology, National Taiwan University Hospital \& National Taiwan University Cancer Center, Taipei, Taiwan \\ ${ }^{7}$ Department of Oncology, Montefiore/Albert Einstein Cancer Center, Bronx, NY, USA \\ ${ }^{8}$ Clinical Trials, Boehringer Ingelheim Taiwan Limited, Taiwan \\ ${ }^{9}$ Epidemiology, Boehringer Ingelheim International GmbH, Ingelheim am Rhein, Germany \\ ${ }^{10}$ Medical Affairs Oncology, Boehringer Ingelheim International GmbH, Ingelheim am Rhein, Germany \\ ${ }^{11}$ Medical Faculty, University Clinic Golnik, University of Ljubljana, Ljubliana, Slovenia \\ *Author for correspondence: Tel.: +43 1910604 1240; maximilian.hochmair@wienkav.at
}

\begin{abstract}
Aim: To assess outcomes in patients with EGFR mutation-positive (Del19, L858R) non-small-cell lung cancer receiving sequential afatinib and osimertinib in a real-world clinical setting. Materials \& methods: In this retrospective, observational, multicenter study, patients $(n=204)$ had T790M-positive disease following first-line afatinib and started osimertinib treatment $\geq 10$ months prior to data entry. Primary outcome was time on treatment. Results: Overall median time on treatment was 27.6 months (90\% Cl: 25.9-31.3), 30.3 months ( $90 \% \mathrm{Cl}: 27.6-44.5)$ in Del19-positive patients and 46.7 months ( $90 \% \mathrm{Cl}: 26.8$-not reached) in Asians. The 2-year overall survival was $78.9 \%$. Conclusion: In real-world clinical practice, sequential afatinib and osimertinib facilitates prolonged, chemotherapy-free treatment in patients with T790M acquired resistance, and is a potentially attractive strategy, especially for Del19-positive tumors.
\end{abstract}

Trial registration number: NCT03370770

First draft submitted: 13 September 2018; Accepted for publication: 9 October 2018; Published online: 19 October 2018

Keywords: afatinib • osimertinib $\bullet$ NSCLC $\bullet$ sequential $\bullet$ T790M

Over the past decade, treatment of EGFR mutation-positive non-small-cell lung cancer (NSCLC) has been revolutionized with the development of EGFR tyrosine kinase inhibitors (TKIs). These agents are associated with improved clinical outcomes and improved tolerability, compared with the previous standard of care, platinum-doublet chemotherapy. Three generations of EGFR TKIs are approved for the first-line treatment of EGFR mutationpositive NSCLC, based on positive Phase III trials: the first-generation reversible TKIs, erlotinib and gefitinib; the second-generation irreversible ErbB family blocker, afatinib; and the third-generation EGFR-wild-type sparing, irreversible EGFR/T790M inhibitor, osimertinib [1].

Recent head-to-head trials have demonstrated that second- and third-generation EGFR TKIs are preferable to first-generation EGFR TKIs in patients with EGFR mutation-positive NSCLC. In the LUX-Lung 7 trial, afatinib conferred significant improvements in progression-free survival (PFS; hazard ratio [HR]: 0.73, 95\% CI: 0.57-0.95; $\mathrm{p}=0.017$ ) and time-to-treatment failure (TTF; HR: 0.73, 95\% CI: $0.58-0.92 ; \mathrm{p}=0.0073$ ) versus gefitinib with a trend toward improved overall survival (OS; HR: $0.86,95 \% \mathrm{CI}: 0.66-1.12 ; \mathrm{p}=0.2580$ ) in patients with 
common EGFR mutations (Del19/L858R) [2,3]. In a similar patient population (but excluding patients with brain metastases), the second-generation TKI, dacomitinib significantly improved PFS (HR: 0.59, 95\% CI: 0.47-0.74; $\mathrm{p}<0.0001$ ) and OS (HR: 0.76, 95\% CI: 0.58-0.99; $\mathrm{p}=0.044$ ) versus gefitinib in the ARCHER-1050 trial [4,5]. In the recent FLAURA trial, osimertinib significantly improved PFS versus gefitinib/erlotinib (HR: $0.46,95 \%$ CI: $0.37-0.57 ; \mathrm{p}<0.001$ ) [6]. OS data are currently immature.

Regardless of which first-line EGFR TKI is chosen, acquired resistance to therapy is inevitable. Therefore, a key consideration when assessing therapeutic choices is the availability of subsequent treatment options following disease progression. The predominant molecular resistance mechanism to gefitinib, erlotinib and afatinib is the emergence of the T790M mutation in exon 20 of $E G F R$, which is present in approximately $50-70 \%$ of tumors at the time of acquired resistance [7-10]. Osimertinib was originally developed to target T790M-positive tumors, and is approved in this setting based on data from the AURA trials (median PFS of 9.6-12.3 months) [9,11,12]. In contrast to first- and second-generation TKIs, targeted therapy options following first-line osimertinib remain limited, due to heterogeneity of resistance mechanisms that are generally not fully understood or currently druggable $[13,14]$. As such, in most patients progressing on osimertinib in everyday clinical practice chemotherapy is the only choice for second-line treatment. There is an argument, therefore, for treating patients with sequential EGFR TKIs, with osimertinib reserved as a second-line option to maximize treatment time on targeted drugs and defer the need for more toxic chemotherapy regimens.

Few data are available that have assessed the cumulative benefit of sequential EGFR TKIs in patients with EGFR mutation-positive NSCLC. In the current observational, global, multicenter study, we have assessed total treatment duration of sequential afatinib and osimertinib in patients with EGFR mutation-positive NSCLC with T790M-aquired resistance who were treated in 'real-world' clinical practice.

\section{Patients \& methods}

\section{Study design}

GioTag was a global, observational study conducted across ten countries (Austria, Canada, Israel, Italy, Japan, Singapore, Slovenia, Spain, Taiwan and the USA; NCT03370770). Medical and electronic health records of consecutive patients treated in a real-world practice who met the following criteria were retrospectively reviewed between 28 December 2017 and 31 May 2018: aged $\geq 18$ years with EGFR-mutated (Del19/L858R) TKI-naive advanced NSCLC who were treated first-line with afatinib, developed the T790M mutation and received secondline osimertinib treatment, and provided written informed consent where required. Patients could have received osimertinib as part of a compassionate use or expanded access program. Patients were excluded if they had received any other first- or second-line treatments, had active brain metastases at the start of either afatinib or osimertinib therapy, or had been treated within a clinical trial. Inclusion was restricted to patients who initiated osimertinib treatment $\geq 10$ months prior to enrollment to avoid early censoring and ensure mature data. A maximum of 15 patients were enrolled per site. Data were sourced either from sites directly approached by the sponsor (38\%) or from electronic health records supplied by Cardinal Health (OH, USA). For quality assurance of the documented patient observations, source data verification was performed on approximately $30 \%$ of included patients.

\section{Outcomes \& assessments}

The primary outcome was time on treatment, defined as the time from the first dose of afatinib to that of the last dose of osimertinib or death. The secondary outcome was the type and proportion of acquired resistance mechanisms after osimertinib treatment. Clinical subgroups were defined based on the characteristics (ethnicity, EGFR mutation, presence of brain metastases and Eastern Cooperative Oncology Group performance status [ECOG PS]) reported at baseline. Treatment interruptions and dose reductions for afatinib and osimertinib were also documented. Sites were requested to provide information on mutational status after osimertinib failure, if available. EGFR mutation and T790M status were determined as per local methodology and practice.

\section{Statistical analysis}

The median time on treatment was predicted to be approximately 24 months, assuming 13-14 months PFS with afatinib and 10-13 months with osimertinib $[2,9,11,15]$. A sample size of 171 patients would give an $80 \%$ likelihood to observe a $90 \% \mathrm{CI}$ of median time on treatment $\leq 10$ months; assuming $10 \%$ of observations would be censored, a sample size of 190 patients was determined. 


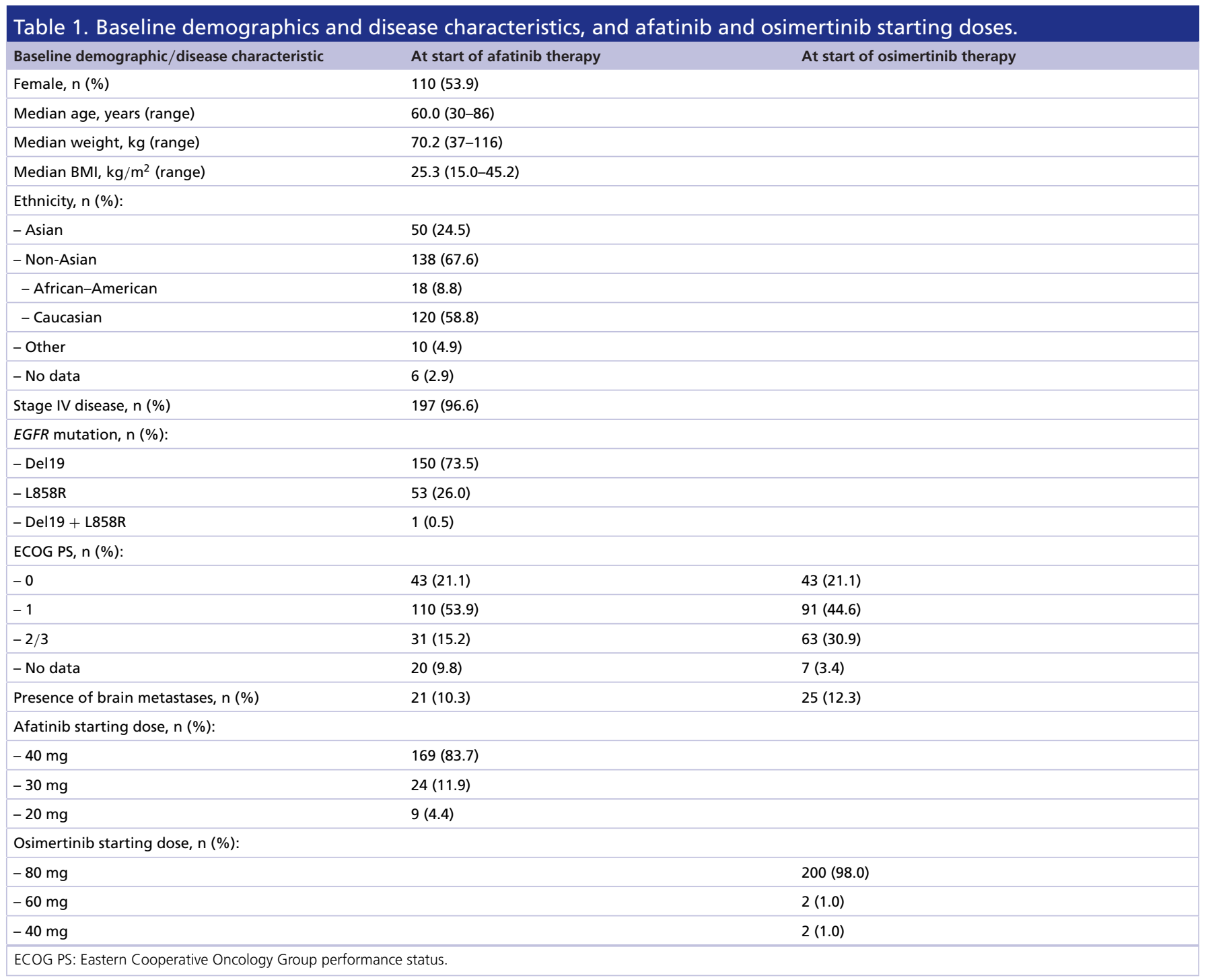

Time on treatment and OS were estimated using the Kaplan-Meier method; for patients still on treatment, time on treatment was censored at the date of data collection. Baseline conditions and demographics, as well as analyses of dose modifications of afatinib and osimertinib were descriptive.

\section{Results}

\section{Patients}

A total of 221 patients were assessed for eligibility and 17 were excluded for protocol violations (Supplementary Figure 1). Baseline characteristics of the 204 patients included in the analysis are shown in Table 1. Patients had diverse ethnicity with 120 (58.8\%) Caucasians, 50 (24.5\%) Asians and 18 (8.8\%) African-American patients; patient enrollment by country is shown in Supplementary Table 1. At the start of afatinib treatment, ECOG PS was 0,1 or $\geq 2$ in $43(21.2 \%), 110(54.2 \%)$ and $31(15.3 \%)$ patients, respectively. A total of 135 patients $(75 \%)$ maintained or even improved ECOG PS during afatinib treatment, and 43 (21.1\%), 91 (44.6\%) and 63 (30.9\%) patients had ECOG PS 0,1 or $\geq 2$ at the start of osimertinib treatment. The proportion of patients with higher ECOG PS was greater at the start of osimertinib treatment. At the start of afatinib treatment, $150(73.5 \%)$ patients had a Del19 mutation and 53 (26.0\%) had the L858R mutation. One patient had both Del19 and L858R. At the start of osimertinib treatment, all patients had a documented T790M mutation. Baseline characteristics according to ethnicity are shown in Supplementary Table 2. Characteristics were generally consistent regardless of ethnicity, although the non-Asian subgroup (Caucasians and African-Americans) included fewer female patients (47.1 vs 


\begin{tabular}{|c|c|c|c|c|c|c|}
\hline $\begin{array}{l}\text { Baseline demographic/disease } \\
\text { characteristic }\end{array}$ & $\begin{array}{l}\text { Overall time on } \\
\text { treatment (months) }\end{array}$ & $\mathrm{p}$-value & $\begin{array}{l}\text { Time on afatinib } \\
\text { treatment }\end{array}$ & $\mathrm{p}$-value & $\begin{array}{l}\text { Time on osimertinib } \\
\text { treatment }\end{array}$ & p-value \\
\hline Overall population & $27.6(25.9-31.3)$ & & $11.9(10.9-12.2)$ & & $14.3(12.8-15.9)$ & \\
\hline Ethnicity: & & & & & & 0.182 \\
\hline - Non-Asian $(n=138)$ & $27.6(24.5-29.2)$ & 0.069 & $11.2(10.4-12.0)$ & 0.001 & $13.8(12.5-15.9)$ & \\
\hline- Asian $(n=50)$ & $46.7(26.8-N R)$ & & $14.0(10.4-16.8)$ & & $19.6(13.5-N R)$ & \\
\hline \multicolumn{7}{|l|}{ Age at start of afatinib therapy: } \\
\hline$-<65$ years $(n=133)$ & $27.6(26.3-30.3)$ & 0.899 & $11.8(10.5-12.1)$ & 0.241 & $14.6(13.4-16.4)$ & 0.556 \\
\hline$-\geq 65$ years $(n=71)$ & $27.6(19.9-44.5)$ & & $12.2(10.5-13.4)$ & & $12.5(9.7-21.4)$ & \\
\hline \multicolumn{7}{|c|}{ EGFR mutation at start of afatinib therapy: } \\
\hline - Del19 $(n=150)$ & $30.3(27.6-44.5)$ & $<0.001$ & $12.6(11.9-13.3)$ & 0.010 & $15.0(13.4-19.6)$ & 0.003 \\
\hline$-\operatorname{L858R}(n=53)$ & $19.1(16.8-25.1)$ & & $10.0(9.0-10.3)$ & & $8.3(6.1-13.5)$ & \\
\hline \multicolumn{7}{|l|}{ Presence of brain metastases: ${ }^{\dagger}$} \\
\hline - Yes $(n=21)$ & $19.4(16.0-N R)$ & 0.037 & $10.4(8.0-13.1)$ & 0.056 & $10.2(7.2-N R)$ & 0.547 \\
\hline - No $(n=183)$ & $28.4(26.7-32.0)$ & & $11.9(11.1-12.2)$ & & $14.3(12.9-15.9)$ & \\
\hline \multicolumn{7}{|l|}{ ECOG PS: ${ }^{\dagger}$} \\
\hline$-0 / 1(n=153)$ & $31.3(27.6-44.5)$ & $<0.001$ & $12.0(11.3-12.6)$ & 0.020 & $15.9(14.3-21.4)$ & $<0.001$ \\
\hline$-\geq 2(n=31)$ & $22.2(16.0-27.0)$ & & $10.4(8.8-12.0)$ & & $9.4(5.6-12.5)$ & \\
\hline
\end{tabular}

66.0\%) and more Del19-positive patients (78.3 vs 62.0\%) than the Asian subgroup. The Asian subgroup included patients of lower bodyweight than the non-Asian subgroup (median weight $61.2 \mathrm{vs} 74.4 \mathrm{~kg}$ ).

Of 204 patients, $169(83.7 \%)$ and $200(98.0 \%)$ patients received starting doses of $40 \mathrm{mg} /$ day afatinib and $80 \mathrm{mg}$ /day osimertinib, respectively (Table 1). The predominant reason for discontinuation of afatinib was progressive disease $(\mathrm{n}=190,93.1 \%$; Supplementary Figure 1). At the time of database lock (29 June 2018), $106(52.0 \%)$ patients had discontinued osimertinib, with progressive disease being the most common reason for discontinuation ( $\mathrm{n}=98,92.5 \%)$; 98 patients were continuing on osimertinib. Half of all patients $(50.5 \%)$ started afatinib therapy before 2016 .

\section{Time on treatment}

After a median follow-up of 28.2 months (range: 14.0-96.8 months), the median time on treatment for sequential afatinib and osimertinib was 27.6 months (90\% CI: 25.9-31.3; Figure 1). Median time on afatinib was 11.9 months (90\% CI: 10.9-12.2) and median time on osimertinib was 14.3 months (90\% CI: $12.8-15.9)$. The median time between stopping afatinib and starting osimertinib was 16 days (interquartile range: 6-33).

Overall time on treatment, and time on afatinib and osimertinib treatment, according to patient subgroups (categorized by ethnicity, age, EGFR mutation type, presence of brain metastases and ECOG PS) are shown in Table 2 and Figure 2. Clinical benefit of sequential afatinib and osimertinib was seen across patient subgroups, with a prolonged median time on treatment reported for Asian patients (overall: 46.7 months; afatinib: 14.0 months; osimertinib: 19.6 months). There was no difference between Caucasian and African-American patients, with 27.6 months for both (Supplementary Table 2). Overall time on treatment (median 30.3 vs 19.1 months), as well as time on afatinib (median 12.6 vs 10.0 months) and time on osimertinib treatment (median 15.0 vs 8.3 months), were longer in patients with Del19 versus L858R mutations. Median time on treatment was also longer in patients with ECOG PS of $0 / 1$ versus $\geq 2$ (overall: 31.3 vs 22.2 months; afatinib: 12.0 vs 10.4 months; osimertinib: 15.9 vs 9.4 months). Patient age had no effect on median time on treatment (median 27.6 months for patients aged $<65$ and those aged $\geq 65$ years). Overall time on treatment was numerically longer in patients without baseline brain metastases versus those with brain metastases (median 28.4 vs 19.4 months; afatinib 11.9 vs 10.4 months and osimertinib 14.3 vs 10.2 months). Of 183 patients with no brain metastasis at baseline, 12 (6.6\%) developed brain metastasis while on afatinib. Of 21 patients with brain metastases at the start of afatinib therapy, $8(38.1 \%)$ were reported to have no brain metastasis at the start of osimertinib therapy. 


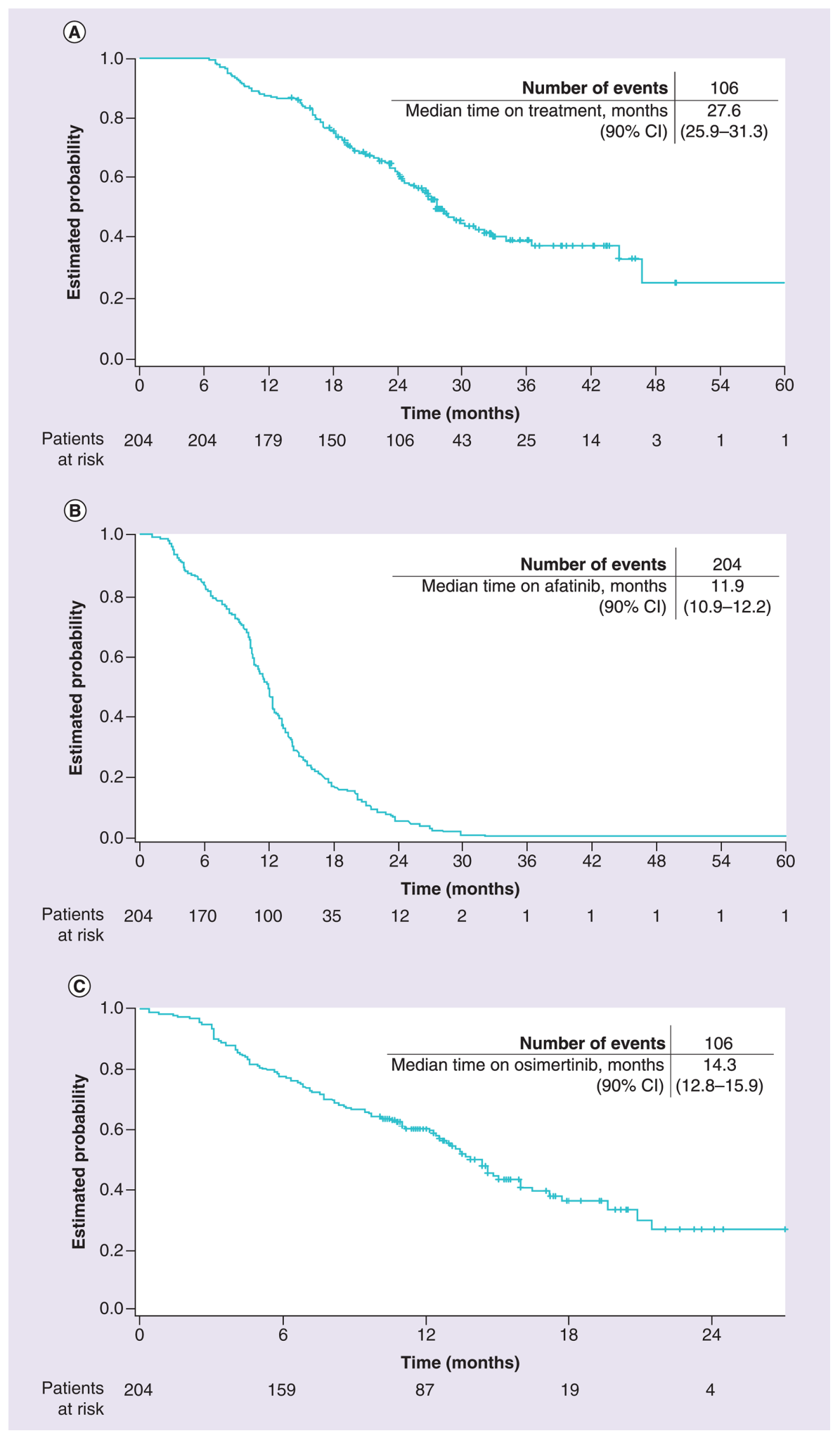

Figure 1. Time on treatment with sequential afatinib and osimertinib in the overall population of 204 patients with EGFR mutation-positive non-small-cell lung cancer. (A) Overall time on treatment with afatinib and osimertinib; (B) time on afatinib treatment; $(C)$ time on osimertinib treatment.

$\mathrm{Cl}$ : Confidence interval. 


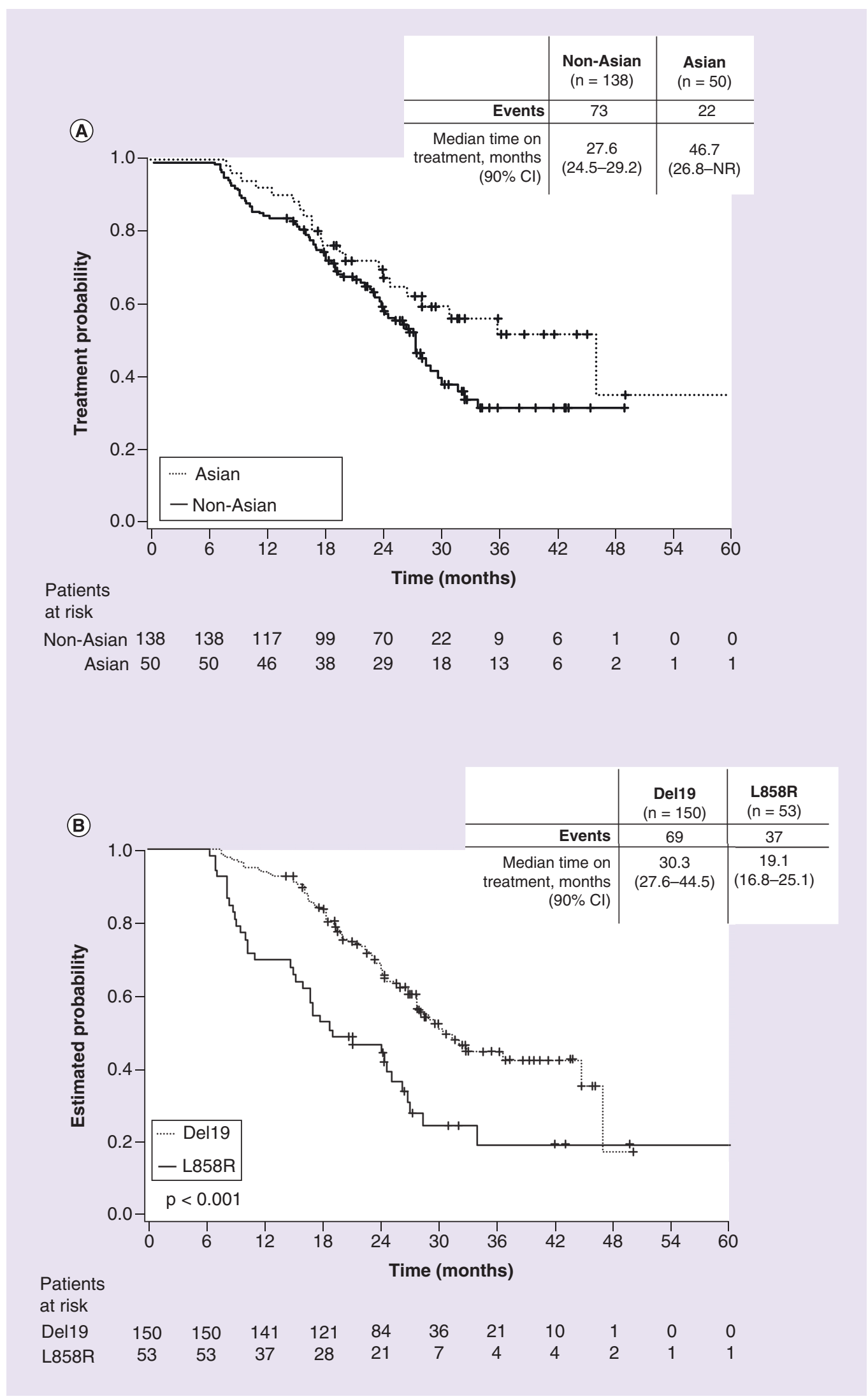

Figure 2. Overall time on treatment with sequential afatinib and osimertinib by subgroup. Time on treatment by (A) ethnicity, (B) type of activating EGFR mutation at baseline, (C) presence or absence of brain metastases at baseline, (D) ECOG PS at baseline.

Cl: Confidence interval; ECOG PS: Eastern Cooperative Oncology Group performance status; NR: Not reached. 


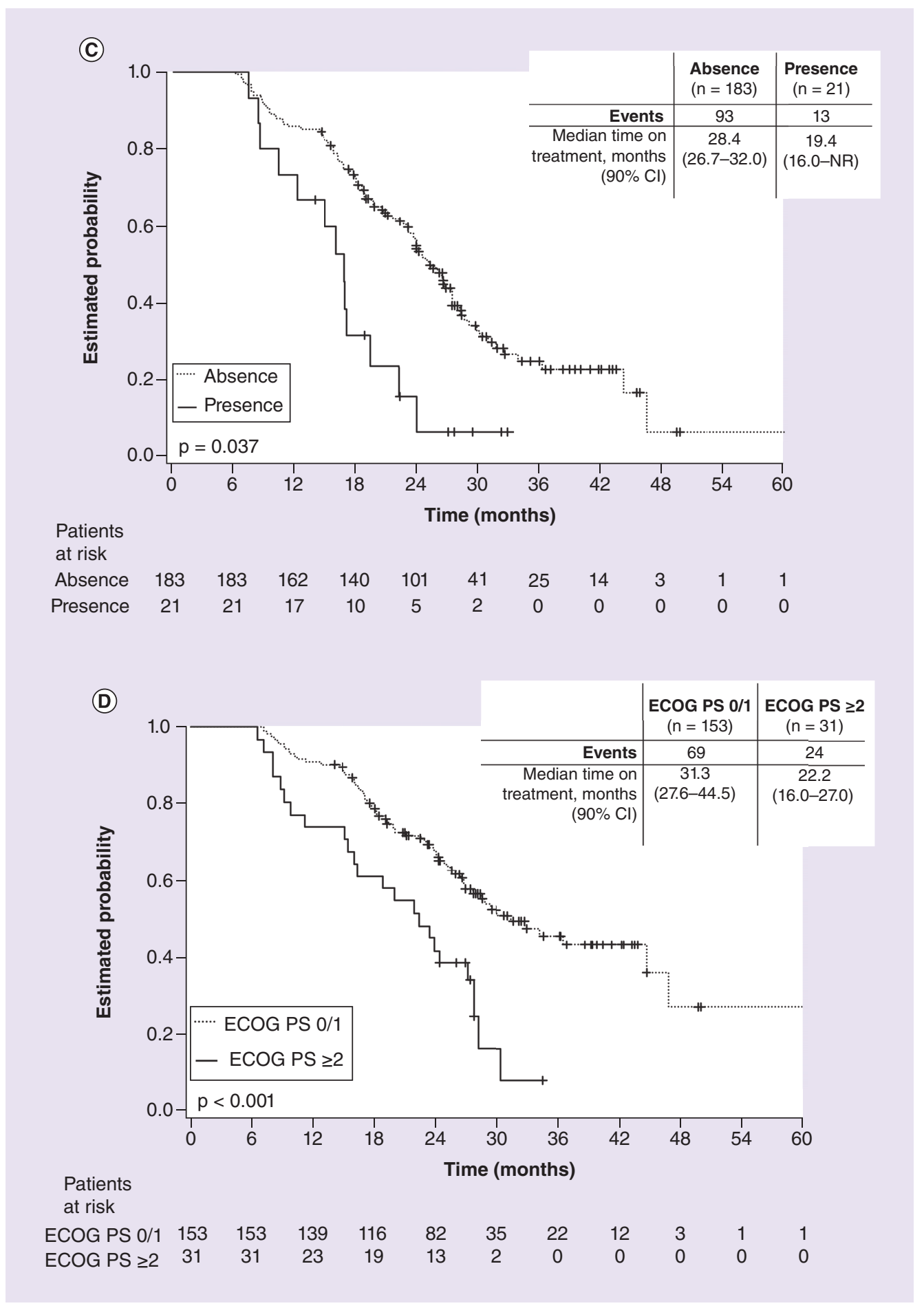

Figure 2. Overall time on treatment with sequential afatinib and osimertinib by subgroup (cont.). Time on treatment by (A) ethnicity, (B) type of activating EGFR mutation at baseline, (C) presence or absence of brain metastases at baseline, (D) ECOG PS at baseline.

Cl: Confidence interval; ECOG PS: Eastern Cooperative Oncology Group performance status; NR: Not reached. 


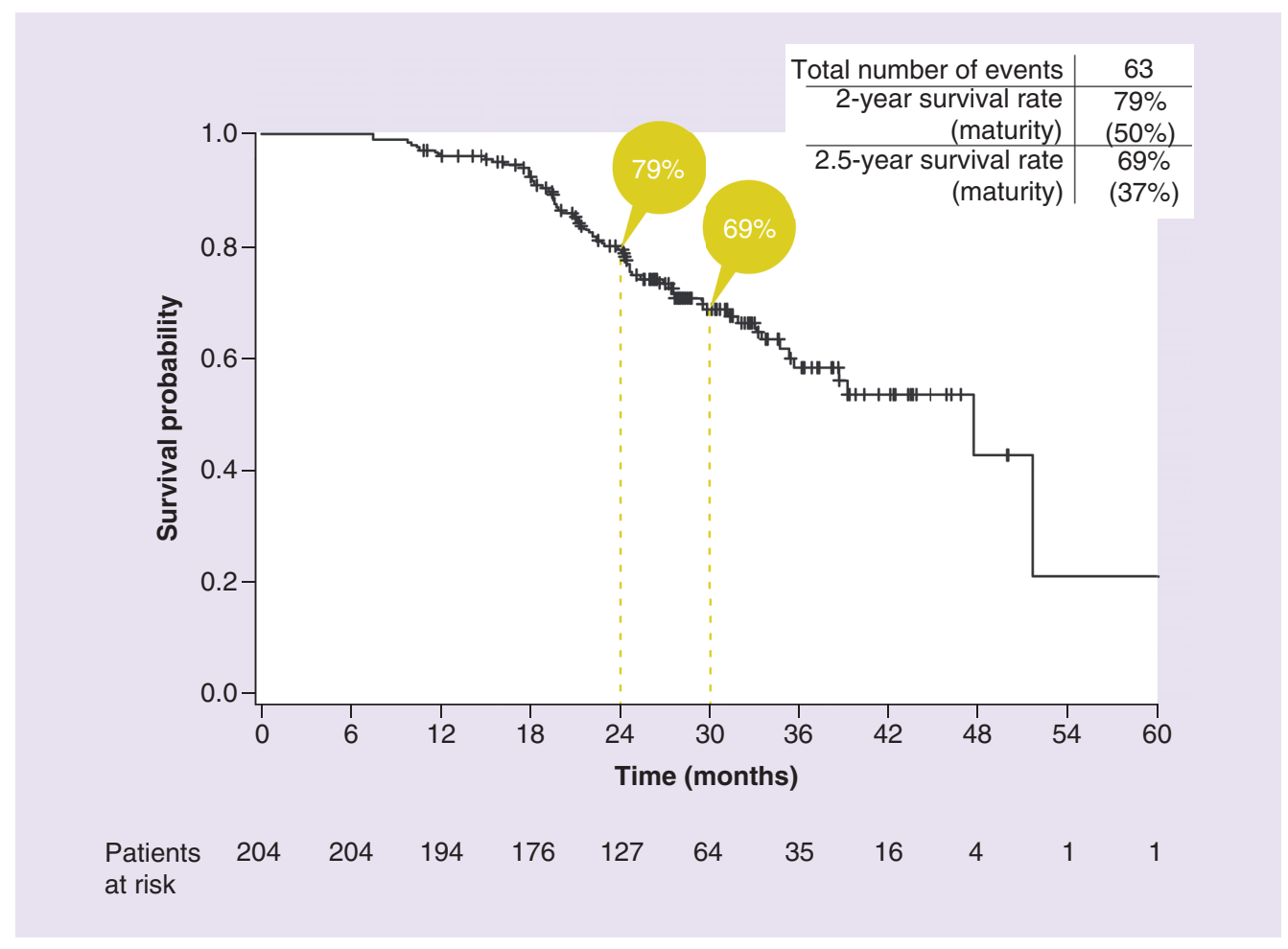

Figure 3. Landmark overall survival at 2 years and 2.5 years in patients treated with sequential afatinib and osimertinib therapy.

\section{Overall survival}

At the time of analysis, 63 patients had died (30.9\%). Therefore, OS analysis was limited to landmarks of 2 and 2.5 years where maturity was 50.0 and $37.1 \%$, respectively. The 2-year OS rate from start of afatinib was $78.9 \%$ (Figure 3). The 2.5-year OS rate was $68.8 \%$. Restricting the analysis to patients with ECOG PS of $0 / 1$, the 2-year landmark was $83.9 \%$ (Supplementary Figure 2).

\section{Mutational status after osimertinib discontinuation}

Of the 106 patients who had discontinued osimertinib, mutational status data were available only for 39 . Of these 39 patients, 27 (69.2\%) had retained both the T790M and EGFR sensitizing mutations, eight (20.5\%) had lost the T790M mutation but retained the EGFR sensitizing mutation, and four (10.3\%) patients had lost the EGFR sensitizing mutation but retained the T790M mutation. No genetic alterations other than the common EGFR mutations (Del19/L858R) and/or T790M were reported. No information on the methodology used was collected.

\section{Discussion}

This observational, global, multicenter study is the first to evaluate outcomes of patients who received first-line afatinib followed by osimertinib. We observed sustained clinical benefit with this regimen in a real-world clinical practice setting in patients with EGFR mutation-positive NSCLC, with a median time on treatment of 27.6 months reported for this broad patient population that also included patients who have not been well represented in prior studies, such as those with ECOG PS $\geq 2(\mathrm{n}=31,15.3 \%)$. Importantly, this clinical benefit was consistent across all patient subgroups, with particularly encouraging results seen for those with Del19-positive disease (median time on treatment 30.3 months) and Asian patients (median time on treatment 46.7 months). These findings suggest that sequential afatinib and osimertinib is an effective treatment option in patients with $E G F R$ mutation-positive NSCLC who acquire T790M, and may be particularly attractive for Del19-positive disease, given that the likelihood of acquiring T790M is approximately 1.5 -fold higher than for L858R-positive disease $[9,10,16-18]$. Around $75 \%$ of patients with Del19-positive disease will acquire T790M resistance, and afatinib as upfront therapy has shown a significantly prolonged OS versus chemotherapy in the Del19-positive patient subgroup [19]. 
This study complements a recent exploratory analysis of LUX-Lung 7, and the LUX-Lung 3 and 6 clinical studies (which compared first-line afatinib with platinum-doublet chemotherapy) [20]. In 37 patients who received osimertinib following afatinib, median duration of treatment on osimertinib was 20.2 months (95\% CI: 12.8-31.5) After 4.7 years, median OS was not reached and the 3 -year OS rate was approximately $90 \%$. In addition, in a recent single-center study $(\mathrm{n}=27)$, the median time on treatment on sequential afatinib and osimertinib was 25.0 months (95\% CI: 20.0-33.0) [10]. Another recent observational study also demonstrated prolonged duration of treatment and survival (67 months) in patients receiving a third-generation EGFR TKI following a first- or second-generation EGFR TKI [21]. While OS data in the current study are immature, and the retrospective nature of the study does not allow for a control arm, the estimated 2-year and 2.5-year OS of 78.9 and 68.8\% are encouraging, particularly noting the possible under-representation of patients who experienced long-term benefit from either drug due to study timelines. While there are little prospective data on the benefits of sequential TKI therapy, prolonged OS has been observed with second-generation TKIs in first-line clinical trial settings. Median OS with afatinib in LUX-Lung 3, with very little subsequent osimertinib use, was 31.6 months [19]. Median OS with dacomitinib in ARCHER-1050 (which excluded patients with brain metastases) was 34.1 months in the intention-to-treat population and 36.7 months in patients who received subsequent osimertinib [5].

A recent analysis concluded that prospective randomized controlled trials in oncology tend to show more effectiveness than seen in the real-world setting, especially if the randomized controlled trials have used surrogate markers such as PFS as end points and if the trial population is directly compared with the overall population, which includes patients not typically enrolled in clinical trials [22]. The prolonged treatment duration with first-line afatinib followed by osimertinib across a broad range of patient subgroups included in this study is therefore encouraging. Indeed, this is consistent with other real-world studies that have demonstrated similar, or better, real-world outcomes with afatinib than observed in clinical trials [23,24]. The median time on osimertinib treatment in this study is longer than the median duration of osimertinib treatment (8.1 months) and median PFS (10.1 months) seen in the AURA-3 clinical trial [11]. These differences might, at least in part, be driven by prolonged treatment beyond RECIST progression in everyday clinical practice. However, the prolonged duration of therapy with osimertinib after afatinib may reflect the observation that afatinib-treated NSCLC cells can show a very high allelic frequency of T790M at the point of acquired resistance (Kohsaka et al. submitted), suggesting that afatinib-resistant tumors are particularly homogenous at the cellular level and therefore may be particularly sensitive to a T790M-directed TKI.

As a real world-based, observational study, this analysis provides data on a broader collective of patients than typically assessed in previous clinical trials of afatinib or osimertinib. For example, 18 (8.8\%) patients were AfricanAmerican, a demographic that was scarcely represented in the LUX-Lung or other EGFR-TKI clinical trials, and 31 $(15.3 \%)$ had an ECOG PS of $\geq 2$, making our results particularly encouraging and applicable to everyday clinical practice. Also, the frequency of patients with Del19-positive tumors (73.5\%) was higher than in the LUX-Lung trials of first-line afatinib (49-58\%) but similar to that observed in the AURA-3 trial of second-line osimertinib (68\%) [2,11,15,25]; probably reflecting the higher frequency of T790M acquired resistance in Del19-positive than L858R-positive tumors $[16,17]$. This observation may also indicate preferential use of afatinib in Del19-positive patients, due to previous OS data [19].

In addition to the particularly notable benefit seen in Asian patients and patients with Del19-positive disease, sequential afatinib and osimertinib conferred prolonged clinical benefit across the range of patient subgroups assessed in this study. Patients with brain metastases is an important subgroup, because CNS involvement is a common feature of EGFR mutation-positive NSCLC [26]. We found a clear time on treatment benefit with afatinib and osimertinib regardless of the presence of brain metastases, supporting previous results indicating that afatinib and osimertinib are effective options in this subgroup $[27,28]$. Of note, although brain imaging was not mandatory and patients with symptomatic brain metastases were excluded, only 12 of $183(6.6 \%)$ patients developed brain metastasis during afatinib therapy, possibly reflecting the ability of afatinib to penetrate the blood-brain barrier [2931]. Compared with Asian and Caucasian patients, little is known about outcomes in African-American patients with EGFR mutation-positive NSCLC, with few such patients enrolled in previous clinical trials of EGFR TKIs. While numbers are small $(\mathrm{n}=18)$, the median time on treatment for African-American patients here was consistent with that seen for the overall population and for Caucasian patients. As might be expected, median time on treatment was significantly shorter for patients with ECOG PS $\geq 2$ versus $0 / 1$. However, it should be noted that in this real-world clinical practice population, patients with PS $\geq 2$, who are rarely included in clinical trials, derived clinical benefit from sequential afatinib and osimertinib treatment, with a median time on treatment of 22.2 months. Consistent 
with previous studies [32,33], age did not appear to have a negative impact on benefit derived from EGFR TKI therapy; overall median time on treatment was 27.6 months in patients $\geq 65$ years old.

The acquisition of T790M is the main mechanism of acquired resistance to afatinib $[9,10,18]$ and osimertinib is a recommended standard second-line therapy for these patients [34]. In contrast, few data are available regarding resistance mechanisms following first-line osimertinib. Resistance mechanisms to later-line osimertinib appear to be heterogeneous. The most predominant mechanism identified to date is the acquisition of the tertiary EGFR mutation, C797S, which is present in approximately $11 \%$ and $22-40 \%$ of cases following first- or later-line administration of osimertinib [35,36]. Other putative resistance mechanisms, such as amplification (e.g., $M E T$, KRAS) or mutation (e.g., MEK1, KRAS, PIK3CA, HER2, BRAF) of other genes, or histological transformation, have been identified but at lower frequency [35-37]. In the present study, only a minority of patients underwent molecular testing following failure of osimertinib, most likely reflecting the lack of available validated targeted treatment options in this setting in routine clinical practice. Moreover, the testing did not identify any druggable alterations. Given the lack of targeted treatment options following osimertinib failure, chemotherapy is the most prevalent treatment option. Indeed, in FLAURA, of the $59 \%$ of patients who received subsequent therapy following osimertinib, 56\% received chemotherapy and 35\% received an off-label EGFR-TKI-based regimen [38].

This study supports the feasibility of a sequencing strategy of afatinib followed by osimertinib in patients with EGFR mutation-positive NSCLC who acquire T790M. The observed turnaround time between discontinuation of afatinib and initiation of osimertinib (median 16 days) indicates that rapid T790M testing in the real-world clinical practice is feasible and that many physicians continue first-line EGFR-TKI treatment until test results are available. Given that the frequency of uptake of subsequent therapy following first-line afatinib is high (71$80 \%$ of patients in LUX-Lung 3, 6 and 7) [2,20], together with the high acquisition rate of T790M in patients receiving afatinib, it is likely that many patients with EGFR mutation-positive NSCLC would be eligible, and fit enough, to receive such a regimen. However, on the other hand, first-line use of osimertinib is supported by highly encouraging efficacy observed in FLAURA [6]. Reserving osimertinib for second-line use could potentially result in some patients (i.e., those whose tumors accrue T790M-independent mechanisms of resistance) never having the opportunity to receive it at all. Furthermore, first-line use of osimertinib is supported by its favorable tolerability profile, with a lower rate of grade $\geq 3$ adverse events than typically observed with first- and second-generation TKIs $[2,4,6]$. Conversely, a treatment strategy involving sequential EGFR TKIs may effectively prolong the overall chemotherapy-free treatment period. Overall, therefore, while there is strong evidence for the use of first-line osimertinib, there remains an argument for considering sequential afatinib and osimertinib, particularly in patients with Del19-positive tumors. The relative benefits and shortcomings of the two strategies remain open to debate at this time and prospective clinical trials are required with OS and quality of life as primary end points.

The main limitations of this study were its retrospective nature and potential for selection bias. Methodological efforts were undertaken to minimize the potential for selection bias, such as including only consecutive patients who fulfilled all of the inclusion criteria and limiting enrollment to a maximum of 15 patients per site. Nevertheless, patients who died on afatinib or were unfit or unwilling for second-line therapy were excluded by the study design; this exclusion of patients who died on first-line afatinib potentially introduced an immortal time bias. However, in the LUX-Lung trials, only approximately $6 \%$ of patients died on first-line afatinib [15,25]. On the other hand, owing to the study timelines (patients must have started second-line osimertinib at least 10 months prior to data collection) and dates of drug approvals and availability (afatinib was approved/reimbursed in participating countries between the second half of 2013 and end of 2014), patients who derived long-term benefit from first-line afatinib (10-12\% in the LUX-Lung trials) had little chance to be enrolled in the study and may therefore have been under-represented. The other main limitation of the study was a lack of a comparator arm, which limits interpretation of the results. The 10-month cutoff for starting osimertinib prior to data entry did not introduce selection bias because there was no specified lower limit on the duration of osimertinib treatment; patients did not have to receive $>10$ months of osimertinib treatment to be included in the study; instead, it was specified as an attempt to maximize maturity of data by reducing risk of early censoring.

\section{Conclusion}

Our results demonstrate the feasibility of sequential afatinib and osimertinib therapy in patients with $E G F R$ mutation-positive NSCLC who acquire T790M. With median time on treatment of 27.6 months in a realworld setting and notably prolonged treatment duration in some patient subgroups, the regimen appears to represent a viable and potentially effective regimen. While prospective studies are needed to fully determine the 
optimum treatment strategy for patients with EGFR mutation-positive NSCLC, this sequential treatment might offer sustained clinical benefit to a substantial number of patients, while avoiding chemotherapy, and warrants consideration.

\section{Summary points}

- Recent randomized, controlled trials have demonstrated that the second-generation ErbB family blocker, afatinib, and the third-generation EGFR TKI, osimertinib, are superior to first-generation TKIs in a first-line setting in patients with EGFR mutation-positive non-small-cell lung cancer (NSCLC).

- There is a lack of validated subsequent treatment options post first-line osimertinib. However, as osimertinib is also a highly effective second-line option in patients with T790M-positive disease, the sequential use of afatinib and osimertinib warrants further assessment as a strategy that might optimize clinical outcomes.

- This international, observational study is the first to evaluate outcomes of patients who received first-line afatinib followed by osimertinib, and collected data between December 2017 and May 2018 from patient medical records.

- Patients had advanced, EGFR mutation-positive (Del19, L858R) NSCLC with T790M-positive disease following first-line afatinib, and must have started osimertinib treatment $\geq 10$ months prior to data entry. Patients with active brain metastases at the start of either therapy were excluded.

- The primary outcome was time on treatment from initiation of afatinib until discontinuation of osimertinib.

- A total of 204 patients were analyzed. Patients were of diverse ethnicity (Asian: $24.5 \%$; non-Asian: $67.6 \%$ ) and $15.3 \%$ had ECOG PS of $\geq 2.73 .5$ and $26.0 \%$ of patients were Del19/L858R-positive.

- Overall, the median time on EGFR-TKI treatment was 27.6 months ( $90 \% \mathrm{Cl}$ : 25.9-31.3). Median time on afatinib and osimertinib was 11.9 months (90\% Cl: 10.9-12.2) and 14.3 months (90\% Cl: 12.8-15.9), respectively.

- In this broad, real-world population, median time on treatment was particularly encouraging in patients with Del19-positive disease (median 30.3 months) and Asian patients (median 46.7 months). Patients with poor prognosis, e.g., those with ECOG PS $\geq 2$ (median 22.2 months) and stable brain metastases (median 19.4 months), also appeared to derive clinical benefit.

- First-line afatinib followed by osimertinib in patients with EGFR mutation-positive NSCLC who acquire T790M is a feasible therapeutic strategy, resulting in a median time on chemotherapy-free treatment of 27.6 months. It may be a particularly attractive option in patients with Del19-positive disease, given the high rate of T790M accrual (around $75 \%$ ) in this subgroup.

\section{Supplementary data}

A video abstract and transcript are available as an accompaniment to this paper. To view the supplementary transcript for this please visit the journal website at: https://www.futuremedicine.com/doi/suppl/10.2217/fon-2018-0711

\section{Acknowledgements}

The authors thank the patients, their families, the investigators and staff who participated in the study. This work was supported by Boehringer Ingelheim. Contract Research Organization support was executed by Parexel. Medical writing assistance, supported financially by Boehringer Ingelheim, was provided by Lynn Pritchard and Jane Saunders of GeoMed, an Ashfield Company, part of UDG Healthcare plc, during the preparation of this article. There is no applicable grant number.

\section{Author's contributions}

The authors were fully responsible for all content and editorial decisions, involved at all stages of manuscript development and have approved the final version.

\section{Data sharing statement}

The datasets generated and analyzed during the study are available from MJ Hochmair on reasonable request.

\section{Financial \& competing interests disclosure}

This study was supported by Boehringer Ingelheim. MJ Hochmair reports personal fees from Speakers honorarium Boehringer Ingelheim, AstraZeneca, and Roche. A Morabito has received honoraria from Boehringer Ingelheim, Roche, AstraZeneca, Pfizer, MSD and Bristol Myers Squibb. D Hao reports research funding and consultancy from Boehringer Ingelheim and Astra Zeneca. RA Soo reports grants and personal fees from Astra Zeneca, personal fees from BMS, Boehringer Ingelheim, Celgene, Lilly, Merck, Novartis, Pfizer, Roche, Taiho and Ignyta. JC-H Yang reports personal fees from Boehringer Ingelheim, Eli Lilly, Roche/Genentech, 
Chugai, Astellas, MSD, Merck Serono, Pfizer, Novartis, Celgene, Merrimack, Yuhan Pharmaceuticals, Bristol Myers Squibb, Ono Pharmaceuticals, Daiichi Sankyo, AstraZeneca, Hansoh Pharmaceuticals and Takeda Pharmaceuticals. B Halmos reports grants and personal fees from Boehringer Ingelheim, Astra Zeneca, Pfizer, Novartis and Takeda, personal fees from Genentech/Roche and grants from Merck. L Wang, A Golembesky and A Märten report employment with Boehringer Ingelheim, TC reports consultancy and honoraria from AstraZeneca, Roche, Pfizer, MSD, Bristol Myers Squibb and Boehringer Ingelheim. C-T Yang and R Gucalp report no competing interests. The authors have no other relevant affiliations or financial involvement with any organization or entity with a financial interest in or financial conflict with the subject matter or materials discussed in the manuscript apart from those disclosed.

Ethical conduct of research

The study was carried out in compliance with the protocol, the principles laid down in the Declaration of Helsinki, in accordance with the International Conference on Harmonization, Harmonized Tripartite Guideline for Good Clinical Practice, Good Epidemiological Practice, Guidelines for Good Pharmacoepidemiology Practice and relevant sponsor Standard Operating Procedures. The study was initiated only after all required legal documentation was reviewed and approved by the respective institutional review board/independent ethics committee and competent authority according to national and international regulations.

Open access

This work is licensed under the Attribution-NonCommercial-NoDerivatives 4.0 Unported License. To view a copy of this license, visit http://creativecommons.org/licenses/by-nc-nd/4.0/

\section{References}

Papers of special note have been highlighted as: • of interest; $\bullet \bullet$ of considerable interest

1. Hirsh V. Turning EGFR mutation-positive non-small-cell lung cancer into a chronic disease: optimal sequential therapy with EGFR tyrosine kinase inhibitors. Ther. Adv. Med. Oncol. 10(1), 1758834017753338 (2018).

2. Park K, Tan EH, O'Byrne K et al. Afatinib versus gefitinib as first-line treatment of patients with EGFR mutation-positive non-small-cell lung cancer (LUX-Lung 7): a Phase 2B, open-label, randomised controlled trial. Lancet Oncol. 17(5), 577-589 (2016).

-. Randomized Phase IIB trial demonstrating improved progression-free survival (PFS) and time-to-treatment failure with afatinib versus gefitinib in patients with $E G F R$ mutation-positive non-small-cell lung cancer (NSCLC).

3. Paz-Ares L. Tan EH, O'Byrne $\mathrm{K}$ et al. Afatinib versus gefitinib in patients with EGFR mutation-positive advanced non-small-cell lung cancer: overall survival data from the Phase IIb LUX-Lung 7 trial. Ann. Oncol. 28(2), 270-277 (2017).

4. Wu YL, Cheng Y, Zhou X et al. Dacomitinib versus gefitinib as first-line treatment for patients with EGFR-mutation-positive non-small-cell lung cancer (ARCHER 1050): a randomised, open-label, Phase 3 trial. Lancet Oncol. 18(11), 1454-1466 (2017).

-• Randomized Phase III trial demonstrating improved PFS with dacomitinib versus gefitinib in patients with EGFR mutation-positive NSCLC.

5. Mok TS, Cheng Y, Zhou X et al. Improvement in overall survival in a randomized study that compared dacomitinib with gefitinib in patients with advanced non-small-cell lung cancer and EGFR-activating mutations. J. Clin. Oncol. 36(22), 2244-2250 (2018).

-• Mature overall survival (OS) data from ARCHER 1050 demonstrating significant OS benefit with dacomitinib versus gefitinib; the first demonstration of OS advantage with a first-line EGFR-TKI in patients with EGFR mutation-positive NSCLC.

6. Soria JC, Ohe Y, Vansteenkiste J et al. Osimertinib in untreated EGFR-mutated advanced non-small-cell lung cancer. N. Engl. J. Med. 378(2), 113-125 (2018).

-• Randomized Phase III trial demonstrating improved PFS with osimertinib versus gefitinib or erlotinib in patients with $E G F R$ mutation-positive NSCLC.

7. Arcila ME, Oxnard GR, Nafa K et al. Rebiopsy of lung cancer patients with acquired resistance to EGFR inhibitors and enhanced detection of the T790M mutation using a locked nucleic acid-based assay. Clin. Cancer Res. 17(5), 1169-1180 (2011).

8. Sequist LV, Waltman BA, Dias-Santagata D et al. Genotypic and histological evolution of lung cancers acquiring resistance to EGFR inhibitors. Sci. Transl. Med. 3(75), 75ra26 (2011).

9. Yang JC, Ahn MJ, Kim DW et al. Osimertinib in pretreated T790M-positive advanced non-small-cell lung cancer: AURA study Phase II extension component. J. Clin. Oncol. 35(12), 1288-1296 (2017).

10. Hochmair M, Schwab S, Burghuber O et al. P2.03-025 prevalence of EGFR T790M mutation in NSCLC patients after afatinib failure, and subsequent response to psimertinib. J. Thorac. Oncol. 12(11), S2137 (2017).

11. Mok TS, Wu YL, Ahn MJ et al. Osimertinib or platinum-pemetrexed in EGFR T790M-positive lung cancer. N. Engl. J. Med. 376(7), 629-640 (2017).

- The Phase III AURA3 study demonstrating significant PFS benefit with osimertinib versus chemotherapy in patients with T790M-positive tumors following progression of erlotinib, gefitinib or afatinib. 
12. Janne PA, Yang JC, Kim DW et al. AZD9291 in EGFR inhibitor-resistant non-small-cell lung cancer. N. Engl. J. Med. 372(18), 1689-1699 (2015).

13. Oxnard GR, Hu Y, Mileham KF et al. Assessment of resistance mechanisms and clinical implications in patients with EGFR T790M-positive lung cancer and acquired resistance to osimertinib. JAMA Oncol. 10.1001/jamaoncol.2018.2969 (2018).

14. Niederst MJ, Hu H, Mulvey HE et al. The allelic context of the C797S mutation acquired upon treatment with third-generation EGFR inhibitors impacts sensitivity to subsequent treatment strategies. Clin. Cancer Res. 21(17), 3924-3933 (2015).

15. Sequist LV, Yang JC, Yamamoto N et al. Phase III study of afatinib or cisplatin plus pemetrexed in patients with metastatic lung adenocarcinoma with EGFR mutations. J. Clin. Oncol. 31(27), 3327-3334 (2013).

16. Ke EE, Zhou Q, Zhang QY et al. A higher proportion of the EGFR T790M mutation may contribute to the better survival of patients with Exon 19 deletions compared with those with L858R. J. Thorac. Oncol. 12(9), 1368-1375 (2017).

17. Matsuo N, Azuma K, Sakai K et al. Association of EGFR Exon 19 deletion and EGFR-TKI treatment duration with frequency of T790M mutation in EGFR-mutant lung cancer patients. Sci. Rep. 6(1), 36458 (2016).

18. Wu SG, Liu YN, Tsai MF et al. The mechanism of acquired resistance to irreversible EGFR tyrosine kinase inhibitor-afatinib in lung adenocarcinoma patients. Oncotarget 7(11), 12404-12413 (2016).

19. Yang JC, Wu YL, Schuler M et al. Afatinib versus cisplatin-based chemotherapy for EGFR mutation-positive lung adenocarcinoma (LUX-Lung 3 and LUX-Lung 6): analysis of overall survival data from two randomised, Phase III trials. Lancet Oncol. 16(2), 141-151 (2015).

20. Sequist L, Wu YS, Schuler M et al. 1349P - Subsequent therapies post-afatinib among patients (pts) with EGFR mutation-positive (EGFRm+) NSCLC in LUX-Lung (LL) 3, 6 and 7. Ann. Oncol. 28(Suppl. 5), v460-v496 (2017).

21. Roeper J, Netcheva M, Lüers A et al. V509 impact on OS and PFS of 2nd and 3rd generation TKI in EGFR mt+ and ALK+ patients: results of the NOWEL network. Oncol. Res. Treat. 40(Suppl. 3), V509 (2017).

22. Lakdawalla DN, Shafrin J, Hou N et al. Predicting real-world effectiveness of cancer therapies using overall survival and progression-free survival from clinical trials: empirical evidence for the ASCO Value framework. Value Health 20(7), 866-875 (2017).

23. Halmos B, Tan EH, Min KL et al. Real-world dose adjustment study of first-line afatinib in pts with EGFR mutation-positive (EGFRm+) advanced NSCLC. J. Clin. Oncol. 36(Suppl.), e21060 (2018).

24. Kim Y, Lee SH, Ahn JS et al. Efficacy and safety of afatinib for EGFR-mutant non-small cell lung cancer, compared with gefitinib or erlotinib. Cancer Res. Treat. doi:10.4143/crt.2018.117 (2018) (Epub ahead of print).

25. Wu YL, Zhou C, Hu CP et al. Afatinib versus cisplatin plus gemcitabine for first-line treatment of Asian patients with advanced non-small-cell lung cancer harbouring EGFR mutations (LUX-Lung 6): an open-label, randomised Phase III trial. Lancet Oncol. 15(2), 213-222 (2014).

26. Hochmair M. Medical treatment options for patients with epidermal growth factor receptor mutation-positive non-small cell lung cancer suffering from brain metastases and/or leptomeningeal disease. Target Oncol. 13(3), 269-285 (2018).

27. Schuler M, Wu YL, Hirsh V et al. First-line afatinib versus chemotherapy in patients with non-small cell lung cancer and common epidermal growth factor receptor gene mutations and brain metastases. J. Thorac. Oncol. 11(3), 380-390 (2016).

28. Goss G, Tsai CM, Shepherd FA et al. CNS response to osimertinib in patients with T790M-positive advanced NSCLC: pooled data from two Phase II trials. Ann. Oncol. 29(3), 687-693 (2018).

29. Hoffknecht P, Tufman A, Wehler T et al. Efficacy of the irreversible ErbB family blocker afatinib in epidermal growth factor receptor (EGFR) tyrosine kinase inhibitor (TKI)-pretreated non-small-cell lung cancer patients with brain metastases or leptomeningeal disease. J. Thorac. Oncol. 10(1), 156-163 (2015).

30. Tamiya A, Tamiya M, Nishihara $\mathrm{T}$ et al. Cerebrospinal fluid penetration rate and efficacy of afatinib in patients with EGFR mutation-positive non-small cell lung cancer with leptomeningeal carcinomatosis: a multicenter prospective study. Anticancer Res. 37(8), 4177-4182 (2017).

31. Girard N. Optimizing outcomes in EGFR mutation-positive NSCLC: which tyrosine kinase inhibitor and when? Future Oncol. 14(11), 1117-1132 (2018).

32. Wu YL, Sequist LV, Tan EH et al. Afatinib as first-line treatment of older patients with EGFR mutation-positive non-small-cell lung cancer: subgroup analyses of the LUX-Lung 3, LUX-Lung 6, and LUX-Lung 7 trials. Clin. Lung Cancer 19(4), e465-e479 (2018).

33. Minuti G, D'Incecco A, Cappuzzo F. Current and emerging options in the management of EGFR mutation-positive non-small-cell lung cancer: considerations in the elderly. Drugs Aging 32(11), 907-916 (2015).

34. Novello S, Barlesi F, Califano R et al. Metastatic non-small-cell lung cancer: ESMO Clinical Practice Guidelines for diagnosis, treatment and follow-up. Ann. Oncol. 27(Suppl. 5), v1-v27 (2016).

35. Ramalingam SS, Yang JC, Lee CK et al. Osimertinib as first-line treatment of EGFR mutation-positive advanced non-small-cell lung cancer. J. Clin. Oncol. 36(9), 841-849 (2018).

- To date, the only study to assess resistance mechanisms to first-line osimertinib in patients with EGFR mutation-positive NSCLC, demonstrating heterogeneous resistance mechanisms. 
36. Tan CS, Kumarakulasinghe NB, Huang YQ et al. Third generation EGFR TKIs: current data and future directions. Mol. Cancer 17(1), 29 (2018).

37. Lim SM, Syn NL, Cho BC et al. Acquired resistance to EGFR targeted therapy in non-small cell lung cancer: mechanisms and therapeutic strategies. Cancer Treat. Rev. 65(1), 1-10 (2018).

38. Planchard D, Boyer M, Lee JS et al. $128 \mathrm{O}$ Osimertinib vs standard of care (SoC) EGFR-TKI as first-line therapy in patients (pts) with untreated EGFRm advanced NSCLC: FLAURA post-progression outcomes. J. Thorac. Oncol. 13(4), S72-S73 (2018). 